\title{
Blood and Synovial Microparticles as Revealed by Atomic Force and Scanning Electron Microscope
}

\author{
Ita Junkar ${ }^{1}$, Vid Šuštar ${ }^{2}$, Mojca Frank ${ }^{3}$, Vid Janša ${ }^{2}$, Apolonija Bedina Zavec ${ }^{4}$, Blaž Rozman ${ }^{3}$, Miran \\ Mozetič $^{1}$, Henry Hagerstrand ${ }^{5}$ and Veronika Kralj-Iglič ${ }^{*}, 2$
}

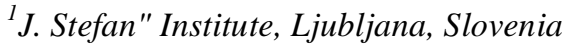 \\ ${ }^{2}$ Laboratory of Clinical Biophysics, Faculty of Medicine, University of Ljubljana, Slovenia \\ ${ }^{3}$ Department of Rheumatology, University Medical Centre Ljubljana, Slovenia \\ ${ }^{4}$ National Institute of Chemistry, Ljubljana, Slovenia \\ ${ }^{5}$ Department of Biology, Abo Akademi University, Abo, Finland
}

\begin{abstract}
Microvesicles which are pinched off the cell membrane can be considered extracellular organelles which mediate interaction between distal cells. They were suggested to play an important role in many diseases including autoimmune disorders, however, standard methods for their assessment have not yet been decided upon while their clinical relevance and the underlying mechanisms are yet unclear. We present a pilot study results involving atomic force microscope (AFM) and scanning electron microscope (SEM) images of the material isolated from peripheral blood of healthy donors and from synovial fluid of patients with psoriatic arthritis and rheumatoid arthritis, which is expected to contain microvesicles. Micrographs reveal in the samples isolated from blood the presence of globular and tubular structures which are most probably microvesicles while the identity of grain-like structures isolated from synovial fluid remains obscure. To the best of our knowledge the AFM and SEM images of the material isolated from synovial fluid are presented for the first time.
\end{abstract}

Keywords: Microvesicles, microparticles, shape, coagulation, antiphospholipid syndrome, thrombosis, cancer.

\section{INTRODUCTION}

Microvesicles [1-4] are formed in the process of budding. In the final stage of this process buds are pinched off the mother membrane and become free to move in the surrounding fluid. They are present in all body fluids and travel with lymph and blood to distal cells. Microvesicles can fuse with distal cells and thereby convey matter and information to these cells. All cells can shed microvesicles, therefore in principle there is no local event in the body.

Microvesicles were found to play an important role in different diseases such as autoimmune diseases [5-8], cardiovascular disorders $[9,10]$ and cancer [11-17]. Thromboembolic events, which are also manifestations of some types of cancer [18] and some autoimmune diseases [19], were associated with presence of microvesicles [20, 21]. Studies of the origin of microvesicles isolated from peripheral blood have shown that the largest pool comes from platelets (around 80\%), erythrocytes contribute around $10 \%$, while the remaining $10 \%$ originates from other cells (T-helper cells, T-supressor cells, monocytes, Blymphocytes, granulocytes and endothelial cells) [22]. Neoplastic cells are prone to shed microvesicles, however, the significant increase of the number of microvesicles found

*Address correspondence to this author at the Laboratory of Clinical Biophysics, Faculty of Medicine, University of Ljubljana, Lipiceva 2, SI-1000 Ljubljana, Slovenia; Tel: +386-41-720-766;

E-mail: veronika.kralj-iglic@fe.uni-lj.si in peripheral blood of patients with certain types of cancer originates from platelets which can be activated by microvesicles from neoplastic cells [15].

Microvesicles and mechanisms underlaying their formation are yet poorly understood, however, it is indicated that they are potentially interesting in diagnosis and treatment of different diseases. For that, a standard protocol should be elaborated and connected to the clinical status of populations as well as of individual persons. For example, analysis of the number and composition of microvesicles could be of help in defining the antiphospholipid syndrome, especially since recognizing the presence of particular antibodies does not yet give a decisive answer. For example, the abundance of endothelial and platelet microvesicles was indicated to correlate more specifically with thrombotic manifestations of antiphospholipid syndrome than antiphospholipid antibody levels alone and might thus provide an additional information for defining the disease [7, $8,23]$. Also detection of microvesicles could be used in conjunction with perceived gold-standards (tissue biopsy, angiography) for the diagnosis of active vasculitis [24] and for assessing the activity of rheumatoid arthritis [25].

It is therefore of interest to better understand microvesiculation and develop methods for assessment of microvesicles for the needs of scientific studies and of clinical practice. Different protocols for isolation of microvesicles can be found in the literature [22, 26-31], usually consisting of centrifugation and washing of the 
sample. To our knowledge the contents of the isolated material obtained by different protocols has not yet been visualized. Here we present the atomic force and scanning electron microscope images of microvesicle-rich plasma and of material isolated from synovial fluid. It is our aim to recognize microvesicles in the material, and determine their morphology and distribution with respect to size. This would enease also interpretation of measurements obtained by other techniques such as flow cytometry.

We present images of the material isolated from peripheral blood of three healthy donors and of the material isolated from synovial fluid of inflammed knee joints of a patient with psoriatic arthritis and a patient with rheumatoid arthritis.

\section{MATERIAL AND METHODS}

\subsection{Donors}

Donors of blood samples were two authors (subject H1: female, 49 years, subject H3: male, 26 years), and a volunteer (subject $\mathrm{H} 2$ : male, 48 years), all with no record of a disease. Donors of synovial fluid samples were a patient with psoriatic arthritis (subject P1; female, 50 years) and a patient with rheumatoid arthitis (subject P2: female, 50 years). Written informed consent was obtained from donors. The research program including the studies on microparticles was approved by National Ethics Committee.

\subsection{Collection of Blood and Synovial Fluid Samples}

After 12 hour fasting, venous blood was collected from donors into $2.7 \mathrm{ml}$ vacutubes (BD Vacutainers), containing $0.109 \mathrm{M}$ trisodium citrate. Synovial fluid was collected by the joint aspiration from actively inflammed knee joints of patients with psoriatic/rheumatoid arthritis into $2.7 \mathrm{ml}$ vacutubes (BC Vacutainer), containing $300 \mu \mathrm{L}$ of $0.109 \mathrm{M}$ trisodium citrate. Immediately after the collection of synovial fluid a further $300 \mu \mathrm{L}$ of $0.109 \mathrm{M}$ trisodium citrate was added to the synovial fluid to prevent clotting as described in [32]. Cells were removed from plasma/synovial fluid by centrifugation for 20 minutes at $1550 \times \mathrm{g}$ and $20^{\circ} \mathrm{C}$. $225 \mu \mathrm{L}$ of microvesicle-rich plasma were used for atomic force microscope imaging.

\subsection{Preparation of Microvesicle-Rich Plasma/Synovial Fluid and Isolation of Microvesicles}

$250 \mu \mathrm{L}$ aliquotes of microvesicle-rich plasma/synovial fluid were used for the isolation of microvesicles. Plasma/synovial fluid was centrifuged for 30 minutes at $17.570 \mathrm{x} \mathrm{g}$ and $20^{\circ} \mathrm{C}$. After the centrifugation, $225 \mu \mathrm{L}$ of microvesicle-poor plasma/synovial fluid supernatant were removed. According to the protocol described by Diamant et al. [22], $25 \mu \mathrm{L}$ of the microvesicle pellet with the remaining plasma/synovial fluid were resuspended in $225 \mu \mathrm{L}$ of phosphate buffered saline (PBS; $137 \mathrm{mM} \mathrm{NaCl}, 2.7 \mathrm{mM} \mathrm{KCl}, 7.8$ $\mathrm{mM} \mathrm{Na}_{2} \mathrm{HPO}_{4} \times 2 \mathrm{H}_{2} \mathrm{O}, 1.5 \mathrm{mM} \mathrm{KH}_{2} \mathrm{PO}_{4}, \mathrm{pH} 7.4$ ), containing $10.9 \mathrm{mM}$ trisodium citrate. After the second centrifugation (30 minutes, $17.570 \mathrm{x} \mathrm{g}$ and $20^{\circ} \mathrm{C}$ ) supernatant $(225 \mu \mathrm{L})$ was removed. For flow cytometric analysis, isolated plasma/synovial microvesicles were resuspended in $75 \mu \mathrm{L}$ of PBS/citrate. For scanning electron microscopy (SEM) analysis, isolated plasma/synovial microvesicles were resuspended in the remaining $\sim 25 \mu \mathrm{L}$ of the supernatant. Aliquotes of 25 $\mu \mathrm{L}$ of resuspended microvesicles were collected into a single $1.5 \mathrm{~mL}$ Eppedorf tube and PBS/citrate was added to the final volume of $250 \mu \mathrm{L}$. The sample was centrifuged again $(60$ minutes, $20.000 \times \mathrm{g}, 20^{\circ} \mathrm{C}$ ). Peleted microvesicles were subsequently prepared for SEM analysis as described in Section 2.5.

\subsection{Atomic Force Microscopy (AFM)}

Microvesicle-rich plasma/isolated synovial microvesicles were fixed to cleaved mica, with atomically smooth surface and incubated on mica for $15 \mathrm{~min}$. After that the samples were rinsed with buffer solution and fixed with $1 \%$ formaldehyde in order to prevent changes in morphology during AFM analysis. Measurements with AFM (Solver PRO, NTMDT, Russia) were done in the tapping mode at room temperature in air. The samples were scanned with standard $\mathrm{Si}$ cantilever (MikroMash) with force constant of $0.2 \mathrm{~N} / \mathrm{m}$, tip radius less then $10 \mathrm{~nm}$ and operating at resonance frequency $184 \mathrm{kHz}$.

In the phase mode imaging, the phase shift of the oscillating cantilever relative to the driving signal is measured. This phase shift can be correlated with specific material properties which depend on the tip-sample interaction. Therefore the phase shift can be used to differentiate areas on a sample with different properties, such as friction, adhesion, and viscoelasticity.

\subsection{Scanning Electron Microscopy (SEM)}

Microvesicles were suspension-fixed in $1 \%$ glutaraldehyde in the PBS/citrate buffer for 60 minutes at $22^{\circ} \mathrm{C}$, postfixed in $1 \% \mathrm{OsO} 4$ in $0.9 \% \mathrm{NaCl}$ for 60 minutes at $22^{\circ} \mathrm{C}$, dehydrated in a graded series of acetone/water $(50$ $100 \%, \mathrm{v} / \mathrm{v})$, critical-point dried, gold-sputtered, and finally examined in a Cambridge Instruments S360 microscope.

\subsection{Flow Cytometry}

Flow cytometric data acquisition and analysis were performed on a flow cytometer (Altra flow cytometer, Beckman Coulter Inc., Fullerton, CA) with a 488-nm water-cooled laser.

Flow-Count fluorospheres of a known concentration (1 $\mu \mathrm{m}$ diameter, $9.7 \times 10^{5} / \mathrm{mL}$ ) from Beckmann-Coulter (Fullerton, CA) were added to the sample to determine the percentage of the measured events. The presence of microvesicles and other particles smaller than $1 \mu \mathrm{m}$ was determined by forward-scatter/side-scatter (FS/SS) dot plot. A minimum of $1 \times 10^{4}$ events was collected for each analysis. For the analysis, $50 \mu \mathrm{L}$ of the sample containing microvesicles and $10 \mu \mathrm{L}$ of fluorospheres were diluted in $150 \mu \mathrm{L}$ of PBScitrate. Beckman Coulter software EXPO32 was used for presentation of results.

\section{RESULTS}

Fig. (1) shows the atomic force micrograph of a sample obtained from microvesicle-rich blood plasma of a healthy donor H1. The height images $(\mathbf{a}, \mathbf{b}, \mathbf{d})$ and the phase image (c) are presented. The globular structures, which differ in elasticity from the surroundings and protrude out of the surface, presumably correspond to microvesicles ranging from 100 to $300 \mathrm{~nm}$ in width and from 30 to $60 \mathrm{~nm}$ in height. 


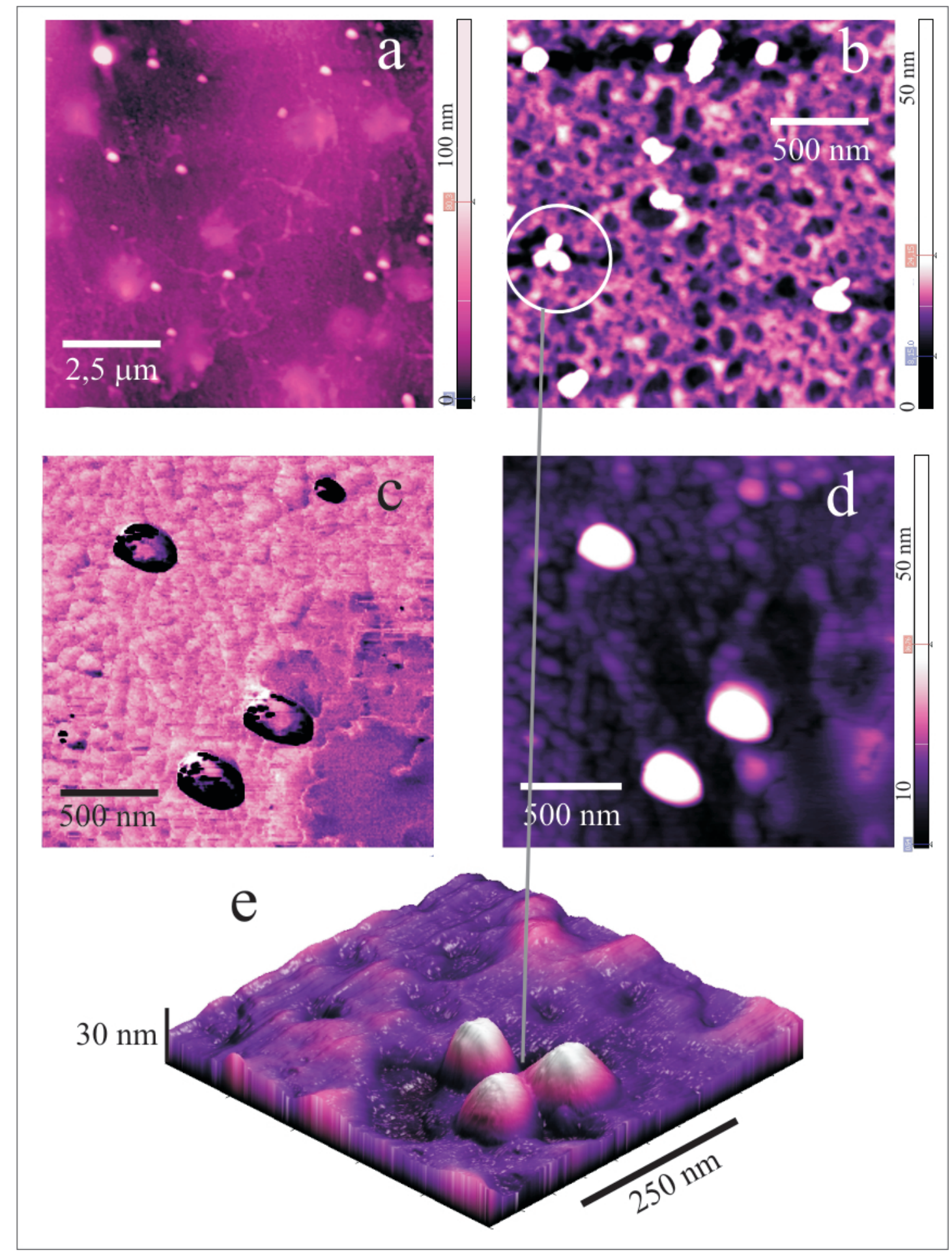

Fig. (1). Atomic force micrograph of the sample of microvesicle-rich blood plasma of healthy donor H1. (a, b, d): the height images, (c): the phase image, (e): enlarged three dimensional representation of the region marked in (b).

Fig. (2) shows the atomic force micrograph of a sample of microvesicle-rich blood plasma of a healthy donor $\mathrm{H} 2$ and a scanning electron micrograph of isolated microvesicles of the donor H3. The height images (a, c) and the phase images (b, d) are presented. Fig. (2a-d) shows many closely packed microvesicles of a similar size (the sample exhibits uniform height about $30 \mathrm{~nm}$ while the estimated lateral dimensions of the structures which are presumably microvesicles is about
$150 \mathrm{~nm}$ ). Boundaries between microvesicles (dark spots) can be seen in the phase images (Fig. 2b, d). The shape of closely packed microvesicles shown in Fig. (2) could be affected by the sample dehydration. The SEM image (e) reveals globular and tubular structures exhibiting shapes which are characteristic for vesicles (membrane-enclosed entities with no internal structure) and are therefore interpreted as microvesicles. 


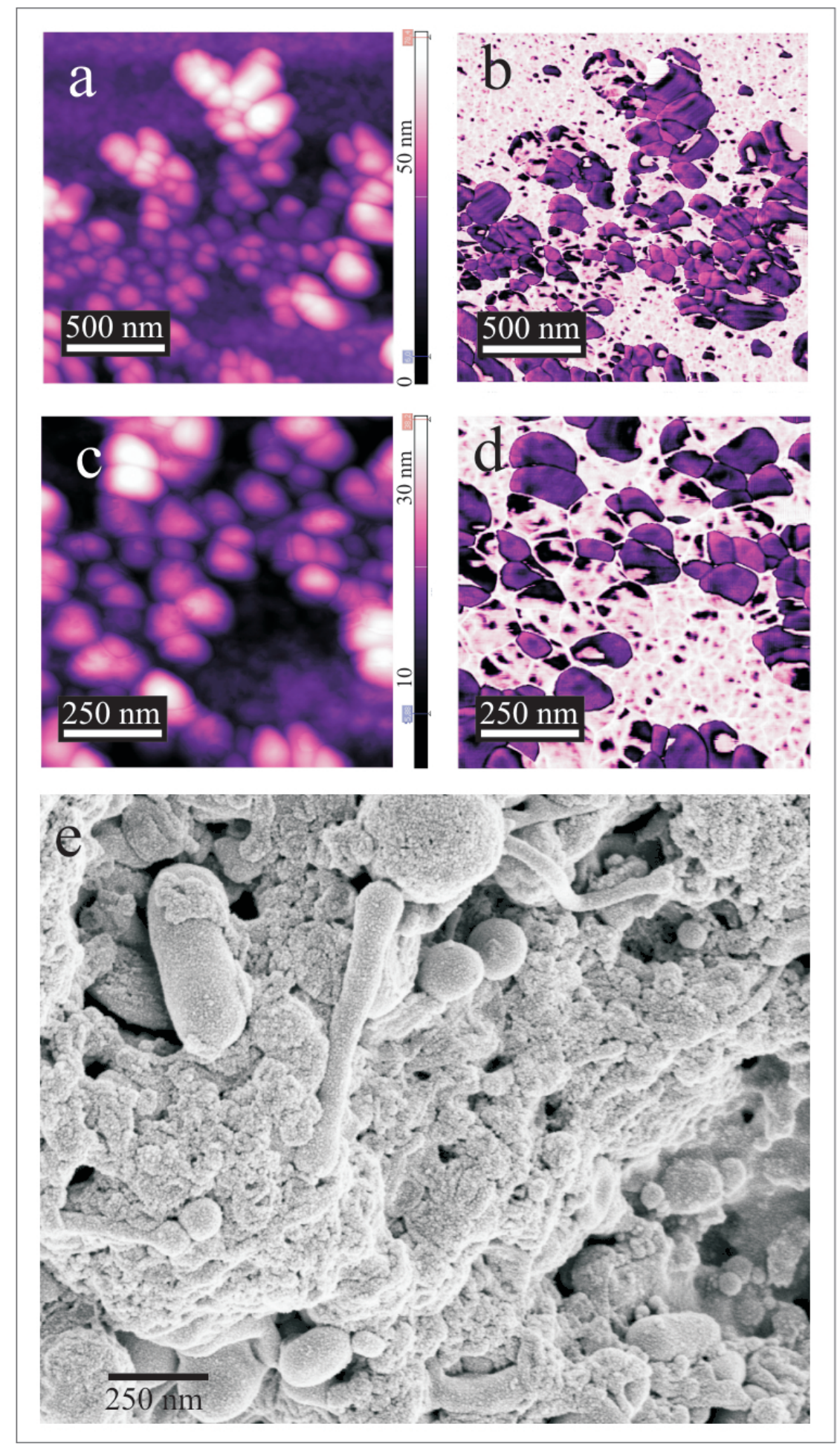

Fig. (2). Atomic force micrograph of the sample of microvesicle-rich blood plasma of healthy donor H2. (a, c): the height images, (b, d): the phase images. (e): the scanning electron micrograph of a sample of microvesicles isolated from peripheral blood of a healthy donor $\mathrm{H} 3$.

Fig. (3) shows the flow cytometric scatter diagrams of FS/SC for the blood samples obtained from healthy donors $\mathrm{H} 1$ and H2 (Figs. 1 and 2). Three regions of events were distinguished. The region R1 corresponds to calibration fluorospheres with diameter $1 \mu \mathrm{m}$. The events in the region $\mathrm{R} 2$ correspond to structures with the same dimensions but different contents of particles yielding different scattering of light. We ascribe these events to the presence of platelets, however, elongated microvesicles may also contribute. Smaller structures ascribed to the region R3 (down to about 50 nanometers) are interpreted as microvesicles and proteinlipid assemblies. Both samples (H1 and $\mathrm{H} 2)$ show similar distributions of events (Table $\mathbf{1}$ ). 

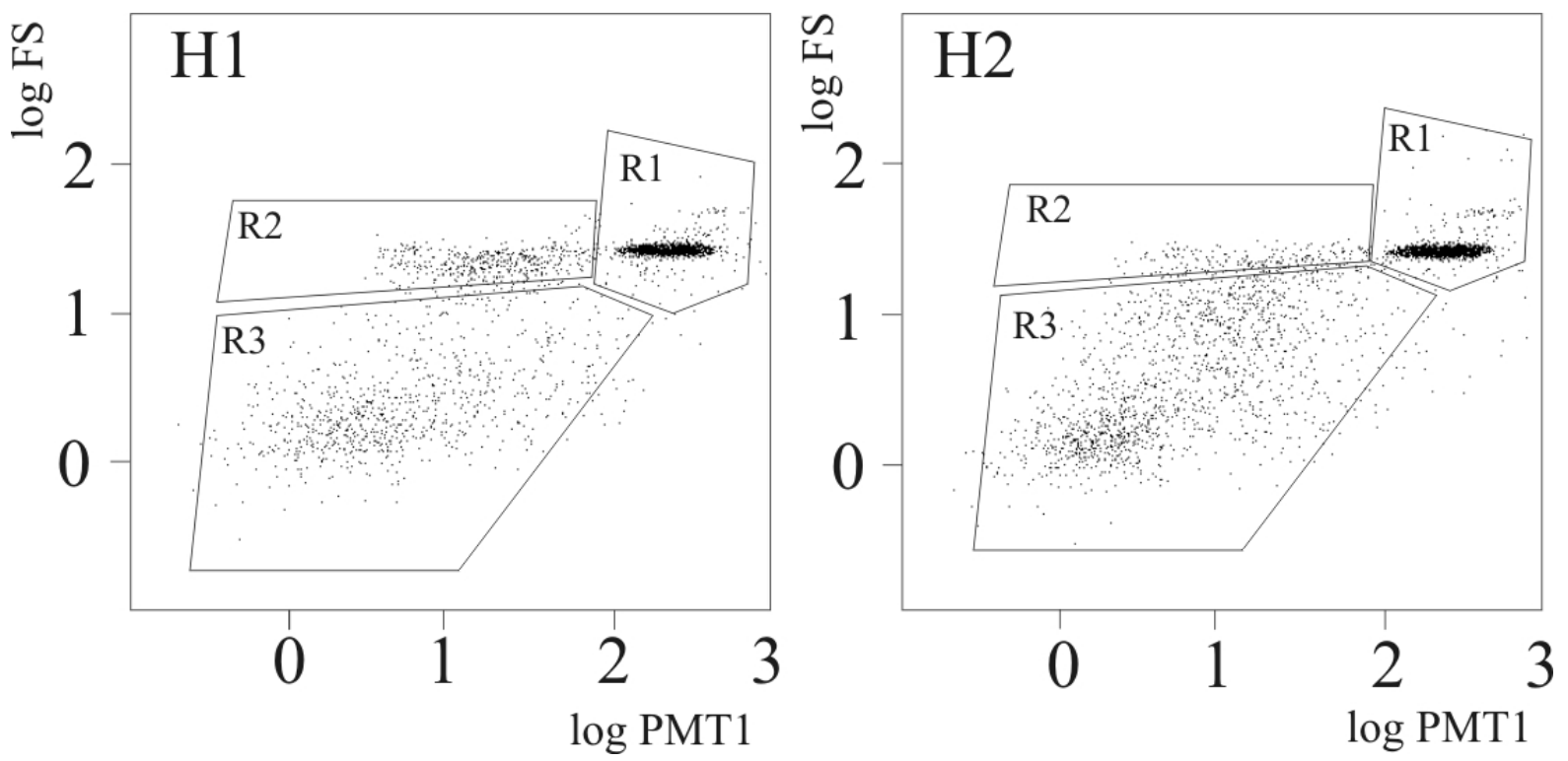

Fig. (3). The flow cytometric scatter diagrams (forward scatter/side scatter) of the material isolated from peripheral blood of healthy donors $\mathrm{H} 1$ and $\mathrm{H} 2$ : calibration fluorospheres with diameter $1 \mu \mathrm{m}(\mathrm{R} 1)$ and particles obtained by the isolation procedure (R2-R3).

Table 1. Distribution of Events Recorded by Flow Cytometry of the Isolated Material Obtained from Peripheral Blood of Healthy Donors (H1 and H2). Events in the Region R1 are Interpreted as Calibration Fluorospheres, Events in the Region R2 are Interpreted as Platelets and Elongated Microvesicles, Events in Region R3 are Interpreted as Microvesicles and Protein-Lipid Assemblies

\begin{tabular}{|c|c|c|c|}
\hline Subject & R1 (\%) & R2 (\%) & R3(\%) \\
\hline \hline H1 & 54 & 19 & 26 \\
\hline H2 & 50 & 17 & 31 \\
\hline
\end{tabular}

Fig. (4) shows the atomic force micrograph of the sample isolated from synovial fluid of the patient with psoriatic arthritis (P1) and the scanning electron micrograph of the sample isolated form synovial fluid of the patient with rheumatoid arthritis (P2). The height images (a, c) and the phase images $(\mathbf{b}, \mathbf{d})$ are presented. The concentration of microvesicles isolated from synovial fluid was much higher than the concentration of microvesicles in the samples obtained by isolation from peripheral blood. Therefore the samples obtained from synovial fluid had to be diluted for AFM analysis. From AFM images we observed grain-like structures of different sizes; their height was about $50 \mathrm{~nm}$. The estimated lateral dimension is between 100 and $150 \mathrm{~nm}$. On the other hand, high density of the sample is favorable for SEM. The SEM image (Fig. 4e) shows many globular structures, which however have more irregular shape compared to microvesicles isolated from blood (Fig. 2e). Grains observed from AFM images (Fig. 4a-d) appear smaller in comparison to microvesicles observed by SEM (Fig. 4e), however the characteristics of the shapes imaged by AFM and SEM seem alike. Furthermore, the grain-like character of shape is revealed in both samples obtained from synovial fluid although they derive from different patients.

\section{DISCUSSION}

Due to their small size, membrane constituents can induce strong curvature in the membrane which forms protrusions and eventually microvesicles. Due to this potential for strongly curving the membrane the pinched off microvesicles are very small and can therefore not be observed directly under the optical microscope. Other techniques are required to reveal their shape. Here we present images obtained by atomic force microscope and scanning electron microscope.

AFM and SEM are appropriate techiques to observe biological material $[33,34]$. By operating AFM in tapping mode we reduced the friction and adhesive force and enabled imaging of soft biological samples. The height of microvesicles determined from AFM was about 25-60 nm which is considerably smaller than their diameter (about 50-200 nm) (Figs.1, 2 and 4). To some extent this could be attributed to AFM tip broadening [35], but most probably due to the collapse of microvesicles during the drying process. This is supported also by SEM analysis, which yields the diameter of comparable dimensions. However, SEM micrographs reveal that the globular structure of microvesicles was conserved reflecting the preparation process which prevents collapse of the microvesicles.

Imaging of microvesicles by AFM in phase mode gives further information about their properties. In phase mode imaging the phase shift of the oscillating cantilever relative to the driving signal is measured and correlated with specific properties of the material, which depend on the cantilever tip-sample interaction. Therefore the phase shift can be used to differentiate areas by friction, adhesion, and viscoelastic- 

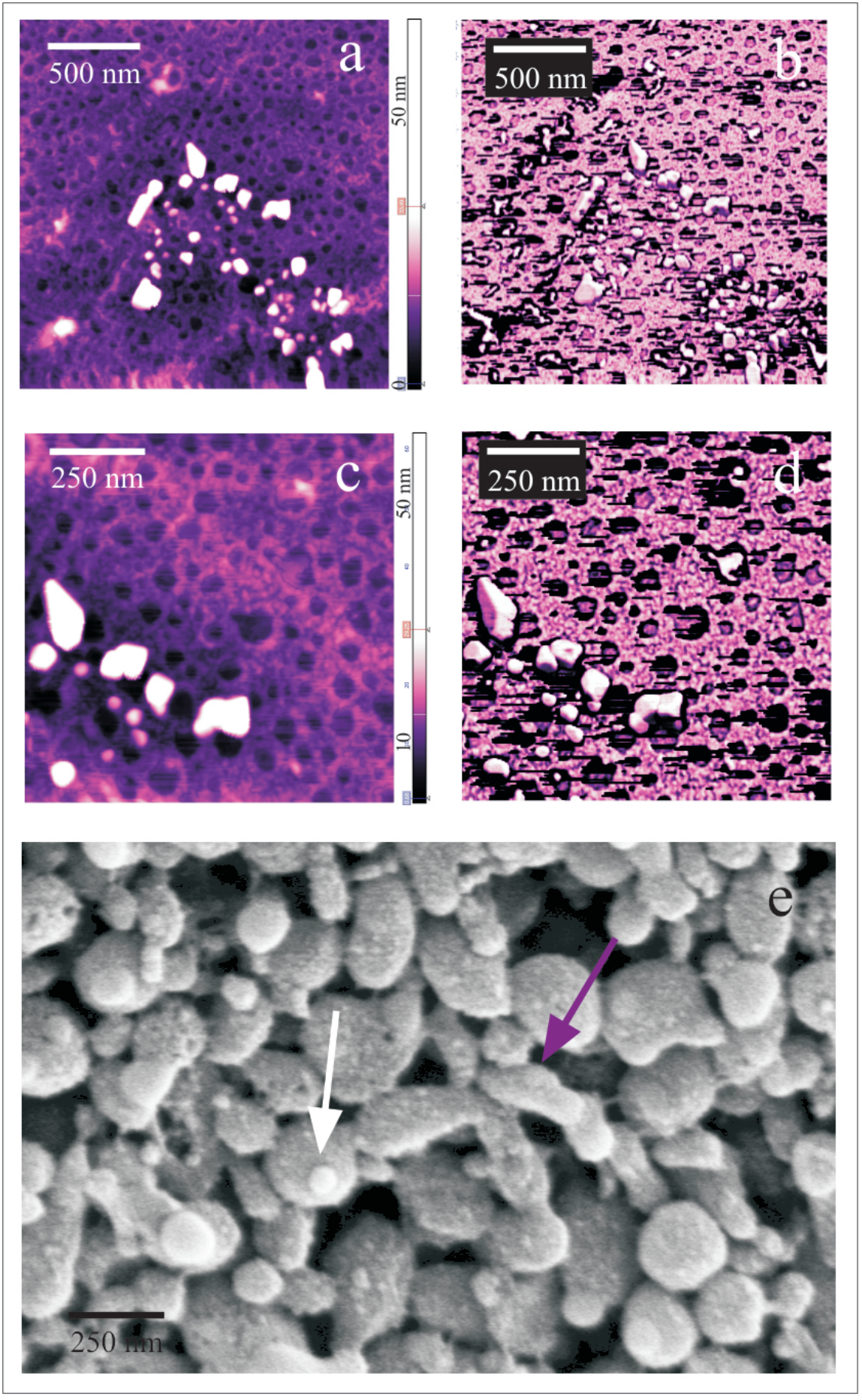

Fig. (4). Atomic force micrograph of the material isolated from synovial fluid obtained from the patient with psoriatic arthritis P1. (a, c): the height images, (b, d): the phase images. (e): the scanning electron micrograph of the material isolated from synovial fluid of the patient with rheumatoid arthritis P2. Globular structures with high degree of symmetry could be microvesicles (white arrow) while more irregular structures could be also protein-lipid assemblies (violet arrow).

ity. In our study this enabled us to distinguish between individual microvesicles by detecting their boundaries (the dark parts in Figs. $2 \mathbf{b}$ and $\mathbf{d}$ and Figs. $\mathbf{3 b}$ and $\mathbf{d}$ ).

In analyzing samples (microvesicle-rich plasma, isolated plasma microvesicles/synovial microvesicles) both techniques revealed presence of globular structures which can be according to their size and shape interpreted as microvesicles. To complement the description of the samples, flow cytometry is used, as this method is able to record a very large number of microvesicles and is therefore convenient for obtaining information on the size distribution. Flow cytometry of the same samples shows that the structures observed by AFM and SEM could correspond to the pool of microvesicles. 
In analyzing the sample obtained from synovial fluid we observed many small structures which could be microvesicles. However, due to somewhat irregular shapes of these grains, which are revealed by the AFM as well as by SEM we cannot decisively claim that all of these structures are microvesicles. Grains could also be formed by assembly of proteins and lipids. It was determined by flow cytometry [32] that most of the synovial grains derive from leukocytes. Further experiments are needed to reveal the morphology of these grains such as the transmission electron microscopy which could give information on the contents of the grains.

Vesicular structures are expected to attain shapes according to the minimum of the free energy of the membrane. The shapes of vesicular structures were observed in artificial systems composed of phospholipid membranes and also studied theoretically [36], and were found to exhibit a high degree of symmetry. In the light of these experiences one can conclude that globular sphere-like and tube-like structures correspond to microvesicles while the identity of the more irregular structures is still unresolved.

It the protocol for isolation of microvesicles which was used in our work, microvesicles are expected to originate from in vivo conditions, however, it is also possible that some of them are formed due to the processing of the sample. Microvesicles could be formed due to the collection of the sample, due to the presence of the anticoagulant in the tubes, due to vesiculation of cells in the time (minutes) between the collection and centrifugation, due to pipetting and during centrifugation. It is yet unclear to what extent these causes contribute to the pool of microvesicles in the sample. The observed samples should therefore be considered as subject to the in vivo conditions as well as of the isolation protocol. It was one of our aims to visualize the sample obtained with the particular protocol [22] used in clinical studies to provide additional information to the data obtained by flow cytometry. Nevertheless, our experience indicates that following the same processing procedure the number of microparticles measured by flow cytometry significantly differs between individuals as well as between groups of patients [37] which indicates that an important (probably the major) pool of microvesicles derives from in vivo conditions.

Microvesicles were found to stimulate cells by transfering surface-bound ligands and receptors between cells [12, 38-40] and deliver biologically important contents such as molecules [12, 41] and infectious particles [42] into cells [16]. Therefore, microvesicles were suggested to play an important role also in mechanisms underlaying autoimmune diseases. It was shown that melanoma as well as ovarian cancer cell - derived microvesicles contain in their membrane Fas ligand and can upon interaction with Fas receptor - carrying $\mathrm{T}$ cells induce apoptosis of $\mathrm{T}$ cells $[11$, 43]. Therefrom, a possible mechanism of tumor progression was suggested by allowing tumor cells to escape immunosurveillance [44]. Based on the evidence on the microvesicle-mediated transport of material between cells, a possible mechanism of platelet destruction in immune thrombocytopenia was suggested. It was hypothesized that microvesicles shed from platelets of patients with immune thrombocytopenia may transfer platelet derived-antigens involved in the formation of anti-platelet antibodies to other cells and thus "expand" the population of target cells that may be affected by anti-platelet antibodies [16]. Increased levels of circulating microparticles were found in patients with acute heparin-induced thrombocytopenia compared with controls [44] and in patients with systemic lupus erythematosus [44], which was connected to thrombotic complications observed in some patients with these disorders $[44,45]$. In most reported disorders including autoimmune diseases, microvesiculation was found to be enhanced. It seems that there is a feed-forward mechanism of microvesicles as indicators as well as promotors of the disorder.

Interplay between microvesiculation and composition of the surrounding solution is of special interest since it may reveal the role of different molecules such as antibodies in the process. Vesiculation was studied in artificial membranes, i.e. giant phospholipid vesicles. These structures are large enough to be observed directly under the phase contrast microscope. Budding of the membrane and pinching off the bud can be induced in a controlled way by changing the temperature or adding substances to the suspension containing vesicles. It was observed that a cofactor in antiphospholipid antibody binding to phospholipids - $\beta 2$ gylcoprotein I - can mediate attractive interaction between negatively charged and neutral membranes [46-48] in the dose-dependent manner [49]. If the effect is strong enough, the bud adheres to the mother membrane and does not become free microvesicle [47]. With this respect, $\beta 2$ glycoprotein I would be anticoagulant which is supported by clinical experience [50]. Also it was found that antiphospholipid antibodies may suppress the mediating effect of $\beta 2$ glycoprotein I [46], thereby exhibiting their role as procoagulants.

In pursue for understanding of autoimmune mechanisms, many works have been devoted to biochemical aspects. However, biophysical methods that have recently been given attention point to nonspecific interactions and their origins [51-53]. We believe that approaches with different points of view will help to improve understanding of microvesiculation and related phenomena.

To conclude, we have observed the material isolated by one of protocols which is used for clinical studies, by AFM and SEM. Both, material isolated from peripheral blood and material isolated from synovial fluid contain structures in the range between 50 and 300 nanometers with characteristic vesicular shapes. However, other shapes are also present, especially in the samples of synovial fluid. Further studies and complementary techniques are required for decisive interpretation of the identity of particles in the isolated material.

\section{REFERENCES}

[1] Diamant M, Tushuizen ME, Sturk A, Nieuwland R. Cellular microparticles: new players in the field of vascular disease? Eur J Clin Invest 2004; 34: 392-401.

[2] Hugel B, Martinez MC, Kunzelmann C, Freyssinet JM. Membrane microparticles: two sides of the coin. Physiology (Bethesda) 2005; 20: $22-7$.

[3] Distler JHW, Pisetsky DS, Huber LC, et al. Microparticles as regulators of inflammation: novel players of cellular crosstalk in the rheumatic diseases. Arthritis Rheum 2005; 52: 3337-48. 
[4] Greenwalt TJ. The how and why of exocytic vesicles. Transfusion 2006; 46: 143-52.

[5] Jy W, Horstman LL, Arce M, Ahn YS. Clinical significance of platelet microparticles in autoimmune thrombocytopenias. J Lab Clin Med 1992; 119: 334-45.

[6] Brogan PA, Shah V, Brachet C, et al. Endothelial and platelet microparticles in vasculitis of the young. Arthritis Rheum 2004; 50: 927-36.

[7] Greer IA. Endothelial cell microparticles and antiphospholipid syndrome: pathologic explanation and diagnostic opportunities. Thromb Haemost 2004; 91: 636-8.

[8] Dignat-George F, Camoin-Jau L, Sabatier F, et al. Endothelial microparticles: a potential contribution to the thrombotic complications of the antiphospholipid syndrome. Thromb Haemost 2004; 91: 667-73.

[9] VanWijk MJ, VanBavel E, Sturk A, Nieuwland R. Microparticles in cardiovascular diseases. Cardiovasc Res 2003; 59: 277-87.

[10] Boulanger CM, Amabile N, Tedgui A. Circulating microparticles: a potential prognostic marker for atherosclerotic vascular disease. Hypertension 2006; 48: 180-6.

[11] Andreola G, Rivoltini L, Castelli C, et al. Induction of lymphocyte apoptosis by tumor cell secretion of FasL-bearing microvesicles. J Exp Med 2002; 195: 1303-16.

[12] Baj-Krzyworzeka M, Majka M, Pratico D, et al. Platelet-derived microparticles stimulate proliferation, survival, adhesion, and chemotaxis of hematopoietic cells. Exp Hematol 2002; 30: 450-459.

[13] Ratajczak MZ. Enhancing effect of platelet-derived microvesicles on the invasive potential of breast cancer cells. Transfusion 2006; 46: 1199-209.

[14] Janowska-Wieczorek A, Wysoczynski M, Kijowski J, et al. Microvesicles derived from activated platelets induce metastasis and angiogenesis in lung cancer. Int J Cancer 2005; 113: 752-60.

[15] Janowska-Wieczorek A, Marquez-Curtis LA, Wysoczynski M, et al. Enhancing effect of platelet-derived microvesicles on the invasive potential of breast cancer cells. Transfusion 2006; 46: 1199209.

[16] Majka M, Kijowski J, Lesko E, et al. Evidence that platelet-derived microvesicles may transfer platelet-specific immunoreactive antigens to the surface of endothelial cells and CD34+ hematopoietic stem/progenitor cells - implication for the pathogenesis of immune thrombocytopenias. Folia Histochem Cytobiol 2007; 45: 27-32.

[17] Whiteside TL. Tumour-derived exosomes or microvesicles: another mechanism of tumour escape from the host immune system? Br J Cancer 2005; 92: 209-211.

[18] Furie B, Zwicker J, LaRocca T, et al. Tissue factor-bearing microparticles and cancer-associated thrombosis. Haem Rep 2005; 1 : 5-8.

[19] Morel O, Jesel L, Freyssinet JM, Toti F. Elevated levels of procoagulant microparticles in a patient with myocardial infarction, antiphospholipid antibodies and multifocal cardiac thrombosis. Thromb J 2005; 3: 15.

[20] Müller I, Klocke A, Alex M, et al. Intravascular tissue factor initiates coagulation via circulating microvesicles and platelets. FASEB J 2003; 17: 476-8.

[21] del Conde I, Shrimpton CN, Thiagarajan P, Lopez JA. Tissuefactor-bearing microvesicles arise from lipid rafts and fuse with activated platelets to initiate coagulation. Blood 2005; 106: 1604-11.

[22] Diamant M, Nieuwland R, Pablo RF, et al. Elevated numbers of tissue-factor exposing microparticles correlate with components of the metabolic syndrome in uncomplicated type 2 diabetes mellitus. Circulation 2002; 106: 2442-7.

[23] Jy W, Tiede M, Bidot CJ, et al. Platelet activation rather than endothelial injury identifies risk of thrombosis in subjects positive for antiphospholipid antibodies. Thromb Res 2007; 121: 319-25.

[24] Brogan PA, Dillon MJ. Endothelial microparticles and the diagnosis of the vasculitides. Intern Med 2004; 43: 1115-9.

[25] Knijff-Dutmer EA, Koerts J, Nieuwland R, et al. Elevated levels of platelet microparticles are associated with disease activity in rheumatoid arthritis. Arthritis Rheum 2002; 46: 1498-503.
[26] Biro E, Nieuwland R, Sturk A. Measuring circulating cell-derived microparticles. J Thromb Haemost 2004; 2: 1843-4.

[27] Dignat-George F, Sabatier F, Camoin-Jau L, Sampol J. Measuring circulating cell-derived microparticles. J Thromb Haemost 2004; 2: 1844-5.

[28] Hugel B, Zobairi F, Freyssinet JM. Measuring circulating cellderived microparticles. J Thromb Haemost 2004; 2: 1846-7.

[29] Jy W, Horstman LL, Jimenez JJ, Ahn YS. Measuring circulating cell-derived microparticles. J Thromb Haemost 2004; 2: 1842-43.

[30] Nomura S. Measuring circulating cell-derived microparticles. J Thromb Haemost 2004; 2: 1847-1848.

[31] Shet AS, Key NS, Hebbel RP. Measuring circulating cell-derived microparticles. J Thromb Haemost 2004; 2: 1848-50.

[32] Berckmans RJ, Nieuwland R, Tak PP, et al. Cell-derived microparticles in synovial fluid from inflamed arthritic joints support coagulation exclusively via a factor VII-dependent mechanism. Arthritis Rheum 2002; 46: 2857-66.

[33] Vesel A, Junkar I, Cvelbar U, et al. Surface modification of polyester by oxygen- and nitrogen-plasma treatment. Surf Interface Anal 2008; 40: 1444-1453.

[34] Drobne D, Milani M, Zrimec A, et al. Focused ion beam/scanning electron microscopy studies of Porcellio scaber (Isopoda, Crustacea) digestive gland epithelium cells. Scanning 2005; 27: 30-4.

[35] Wilson DL, Kump KS, Eppell SJ, Marchant RE. Morphological restoration of atomic force microscopy images. Langmuir 1995; 11 : 265-72.

[36] Iglič A, Babnik B, Gimsa U, Kralj-Iglič V. On the role of membrane anisotropy in beading transition of undulated tubular membrane structures, J Phys A: Math Gen 2005; 38: 8527-36.

[37] Janša R, Šuštar V, Frank M, et al. Number of microvesicles in peripheral blood and ability of plasma to induce adhesion between phospholipid membranes in 19 patients with gastrointestinal diseases. Blood Cells Mol Dis 2008; 41: 124-32.

[38] Ratajczak J, Wysoczynski M, Hayek F, et al. Membrane-derived microvesicles (MV): important and underappreciated mediators of cell to cell communication. Leukemia 2006; 20: 1487-95.

[39] Krzyworzeka M, Reca R, Turner AR, et al. Platelet-derived microparticles bind to hematopoietic stem/progenitor cells and enhance their engraftment. Blood 2001; 98: 3143-9.

[40] Rozmyslowicz T, Majka M, Kijowski J, et al. Platelet- and megakaryocyte- derived microparticles transfer CXCR4 receptor to CXCR4-null cells and make them susceptible to infection by X4HIV. AIDS 2003; 17: 33-42.

[41] Ratajczak J, Miekus K, Kucia M, et al. Embryonic stem cellderived microvesicles reprogram hematopoietic progenitors: evidence for horizontal transfer of mRNA and protein delivery. Leukemia 2006; 20: 847-56.

[42] Pelchen-Matthews A, Raposo G, Marsh M. Endosomes, exosomes and Trojan viruses. Trends Microbiol 2004; 12: 310-6.

[43] Abrahams VM, Straszewski SL, Kamsteeg M, et al. Epithelial ovarian cancer cells secrete functional Fas ligand. Cancer Res 2003; 63: 5573-81.

[44] Warkentin TE, Hayward CP, Boshkov LK, et al. Sera from patients with heparin-induced thrombocytopenia generate platelet-derived microparticles with procoagulant activity: an explanation for the thrombotic complications of heparin-induced thrombocytopenia. Blood 1994; 84: 3691-3699.

[45] Pereira J, Alfaro G, Goycoolea M, et al. Circulating plateletderived microparticles in systemic lupus erythematosus : association with increased thrombin generation and procoagulant state. Thromb Haemost 2006; 95: 94-9.

[46] Ambrožič A, Čučnik S, Tomšič N, et al. Interaction of giant phospholipid vesicles containing cardiolipin and cholesterol with B2glycoprotein-I and anti-ß2-glycoprotein-I antibodies. Autoimmun Rev 2006; 6: 10-5.

[47] Urbanija J, Tomšič N, Lokar M, et al. Coalescence of phospholipid membranes as a possible origin of anticoagulant effect of serum proteins. Chem Phys Lipids 2007; 150: 49-57. 
[48] Urbanija J, Babnik B, Frank M, et al. Attachment of B2glycoprotein I to negatively charged liposomes may prevent the release of daughter vesicles from the parent membrane. Eur Biophys J 2008; 37: 1085-95.

[49] Frank M, Manček-Keber M, Kržan M, et al. Prevention of microvesiculation by adhesion of buds to the mother cell membrane a possible anticoagulant effect of plasma. Autoimmun Rev 2008; 7: 240-5.

[50] Brighton TA, Hogg PJ, Dai YP, et al. Beta 2-glycoprotein I in thrombosis: evidence for a role as a natural anticoagulant. Br J Haematol1996; 93: 185-94.
[51] Bohinc K, Iglič A, May S. Interaction between macroions mediated by divalent rod-like ions. Europhys Lett 2004; 68: 494-500.

[52] May S, Iglič A, Reščič J, et al. Bridging like-charged macroions through long divalent rod-like ions. J Phys Chem B 2008; 112: $1685-92$.

[53] Urbanija J, Bohinc K, Bellen A, et al. Attraction between negatively charged surfaces mediated by spherical counterions with quadrupolar charge distribution. J Chem Phys 2008; 129: 105101.

(C) Junkar et al.; Licensee Bentham Open.

This is an open access article licensed under the terms of the Creative Commons Attribution Non-Commercial License (http://creativecommons.org/licenses/by-nc/3.0/) which permits unrestricted, non-commercial use, distribution and reproduction in any medium, provided the work is properly cited. 